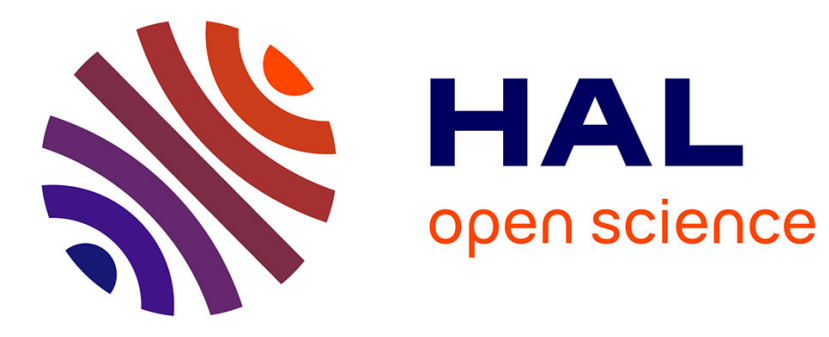

\title{
Dense granular dynamics analysis by a domain decomposition approach
}

Vincent Visseq, Alexandre Martin, Damien Iceta, Emilien Azéma, David Dureisseix, Pierre Alart

\section{- To cite this version:}

Vincent Visseq, Alexandre Martin, Damien Iceta, Emilien Azéma, David Dureisseix, et al.. Dense granular dynamics analysis by a domain decomposition approach. Computational Mechanics, 2012, 49 (6), pp.709-723. 10.1007/s00466-012-0699-5 . hal-00689838v2

\section{HAL Id: hal-00689838 \\ https://hal.science/hal-00689838v2}

Submitted on 1 Nov 2016

HAL is a multi-disciplinary open access archive for the deposit and dissemination of scientific research documents, whether they are published or not. The documents may come from teaching and research institutions in France or abroad, or from public or private research centers.
L'archive ouverte pluridisciplinaire HAL, est destinée au dépôt et à la diffusion de documents scientifiques de niveau recherche, publiés ou non, émanant des établissements d'enseignement et de recherche français ou étrangers, des laboratoires publics ou privés. 


\title{
Dense granular dynamics analysis by a domain decomposition approach
}

\author{
V. Visseq ${ }^{(1)}$, A. Martin(1) ${ }^{(1)}$ D. Iceta ${ }^{(1)}$, E. Azema $^{(1)}$, \\ D. Dureisseix ${ }^{(2)}$, P. Alart ${ }^{(1)}$
}

(1) Laboratoire de Mécanique et Génie Civil (LMGC), Université Montpellier 2 / CNRS UMR 5508, Place E. Bataillon, F-34095 Montpellier, France, Tel.: 334671439 89, Fax: 334671439 23, \{Vincent.Visseq, Alexandre.Martin, Damien.Iceta, Emilien.Azema, Pierre.Alart $@ @ u n i v-m o n t p 2 . f r$

(2) Laboratoire de Mécanique des Contacts et des Structures (LaMCoS), INSA Lyon / CNRS UMR 5259, 18-20 rue des Sciences, F-69621 Villeurbanne CEDEX, France, Tel.: 334724383 65, Fax: 334788909 80, David.Dureisseix@insalyon.fr

\begin{abstract}
In this article a domain decomposition approach is combined with the nonsmooth contact dynamics approach for analysing the global behaviour and the micromechanical structure of large-scale dense granular systems. Previously introduced and theoretically investigated, this method is herein investigated precisely on two aspects: the properties of the interface operators, especially when applied to the corners of the subdomains, and the influence of the substructuring on the solution of a mechanical test. Such topics are specific to the dense granular systems characterized by the discreteness and the nonsmoothness of their behavior.

This article has been published in its final form as: V. Visseq, A. Martin, D. Iceta, E. Azema, D. Dureisseix, P. Alart, Dense granular dynamics analysis by a domain decomposition approach, Computational Mechanics 49(6):709-723, Springer, 2012. The final publication is available at Springer via http://dx.doi.org/10.1007/s00466-012-0699-5
\end{abstract}

Keywords: domain decomposition method; nonsmooth contact dynamics; discrete elements; frictional contact; granular packing; LMGC90

PACS: $\quad$ 45.70.Cc; 83.10.Rs; 83.80.Fg

MSC: $65 \mathrm{Y} 05 ; 70 \mathrm{E} 55$

\section{Introduction}

In connection with a domain decomposition strategy, the granular dynamics reveals two main features: discreteness and nonsmoothness.

The non-overlapping decomposition of a granular domain is all the more delicate since such a medium is a non-structured discrete system. Contrary to 
trusses or tensegrity structures studied in [36] for which an elementary pattern may be defined during the whole process, a granular system involves a permanent evolution of the connectivity of the particles, specially when granular flows occur. Consequently a box-like partitioning insuring a locality of data, useful for a parallel implementation, provides two possible approaches. A primal strategy leads to a 'non-perfect' interface between the subdomains made of nonsmooth interactions. Because such a method is a simple algebraic partition of the equations and is easy to implement, it has been applied to an industrial problem, the simulation of railway ballasts [21]. However when some large rigid bodies constitute the boundaries of several subdomains of the system (as the sleepers on a railway track), the size of the interface increases drastically. The dual strategy is less intuitive because it requires to split the grains at the interface. Contrary to the primal approach the interface behavior is now 'perfect', in the sense that only linear equations are describing it (local equilibrium and velocity continuity). Indeed we have to glue the interface grains by adding a new, but linear, equation which modifies strongly the global nonlinear (nonsmooth) solver and which complicates the implementation. However this dual approach has two advantages: (i) the occurrence of large rigid bodies do not perturb the size of the interface; (ii) the perfect boundary of each subdomain should allow to introduce an automatic homogenization process to switch possibly from a discrete model toward a continuous model. This second approach is detailed in the following.

Once the sub-structuring has been performed, a nonsmooth solver has to be combined with the domain decomposition strategy. The nonsmooth relations are derived from the NonSmooth Contact Dynamics (NSCD) approach which is well suited to the simulation of granular systems. NSCD or Contact Dynamics in short, has been developed by J. J. Moreau and M. Jean over the last two decades $[24,34]$. It is suited to many applications but has proven to be particularly useful when collections of rigid or deformable bodies are packed together in a dense assembly and subjected to dynamic loading deformations. Numerical simulations have to be performed using a fully implicit resolution of the contact impulses. This allows us to deal properly with nonlocal momentum transfers involved in multiple collisions, contrary to classical molecular dynamics schemes that consider the system evolution as a succession of binary collisions. The approach proposed by Cundall [14], inspired from the molecular dynamics, as the event-driven methods, requires very short time steps for accounting for the successive collisions and such a strategy leads to a prohibitive computational cost, especially for the dense granulates with a large number of simultaneous collisions. The NSCD approach refers to a time-stepping method that requires at each time step the solution of nonsmooth equations by an iterative solver. The computational cost may be quite high, but the gain is substantial. Simulations of very large granular systems can range from $10 \mathrm{~m}$ of a ballast railway submitted to cyclic dynamic loading, to the behavior of the Nîmes arena and Arles aqueduct (France) subjected to seismic loading, which are examples of two challenges in computational mechanics.

The Domain Decomposition Methods (DDM) in the context of multiprocessor computations are well established from theoretical and practical standpoints when dealing with a linear system derived from a discretization of a continuous problem [29]. For nonlinear continuous problems the DDM seems to be efficient when it is used only to solve an intermediate linear problem embedded in 
an iterative process as a Newton type method [15]. Unfortunately the simulation of the granular systems with nonsmooth interactions between grains does not use such a nonlinear solver and the combination with a DDM has to be rethought. Indeed the NonLinear Gauss-Seidel (NLGS) algorithm may be considered as the generic nonsmooth solver because it allows to embed a large range of interaction laws such as adhesion, cohesion, capillarity... without modifying deeply the algorithm. In line with the NSCD approach, the velocity-impulse formulation is extended herein to a multidomain reformulation preserving the generic algorithm. More precisely the multidomain reformulation is based on a FETI-type approach where the subdomains are 'glued' by Lagrange multipliers which are inter-domain forces. This choice is made in accordance with the NSCD approach where the impulses are privileged variables. The so-called NonSmooth Contact Domain Decomposition (NSCDD) solving method consists of a two-stage algorithm. One of these stages recovers the generic NLGS method applied subdomain per subdomain in conjunction with the NSCD formulation; for details about convergence, refer to [25]. This generic algorithm is presented in Section 2 and a theoretical study of the convergence is developed in [4].

The DDM introduces different types of interface according to their dimension. For a three-dimensional continuous problem, we distinguish facets, edges and corners. Specific strategies are developed for dealing with the corners resulting from the domain decomposition of structures discretized by a finite element method $[19,30]$. For discrete systems the distinction is less clear and we have developed in [3] the concept of 'weak' interfaces in the context of static problems solved by a LATIN type method. We investigate in Section 3 the features of the interface problem solved at the second stage of the generic algorithm when some grains, located at the corners, are connected to more than two subdomains.

Finally the evaluation of the efficiency of a new multidomain solver in comparison with a previous monodomain one is a difficult topic because a dense granular system is an evolutive nonsmooth problem leading to a large multiplicity of solutions [32]. Consequently we have not relevant error estimates as underlined in [24]. Only the quality of the computation may be appreciated using a set of qualitative indicators as presented in Section 4 for studying the global behavior and the micromechanical structure of a granular sample submitted to a biaxial test. A time consuming analysis is performed on a Sequential Multidomain implementation in order to estimate the CPU time gain that we can expect from a multiprocessing implementation on a distributed memory architecture. The present approach has been implemented into the LMGC90 platform [16].

\section{Dual domain decomposition method for gran- ular systems}

\subsection{Reference problem}

When a time-stepping scheme is used, we denote known quantities at the beginning of the time slab $\left[t_{i}, t_{i+1}\right]$ with a superscript $(-)$; the quantities at the end of the time slab (without a superscript) have to be determined. 


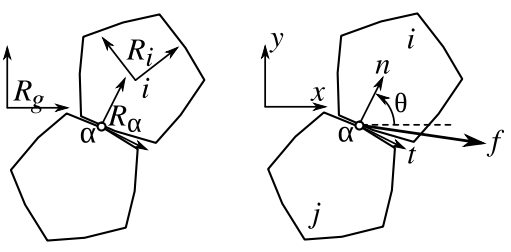

Figure 1: Coordinate basis; $R_{g}$ : global coordinate basis (for interface quantities), $R_{i}$ : local (related to the grain $i$ ) coordinate basis (for grain dynamics), $R_{\alpha}$ : local (related to an interaction $\alpha$ between two grains) coordinate basis (for interactions). Details of the local contact frame $(n, t)$ at a contact $\alpha$ between two touching particles $i$ and $j$.

Grain nonsmooth dynamics. Considering a rigid model for the grains, the dynamics of the granular medium is written as the following vector equation $[34]$

$$
M V-R=F^{d}
$$

where the prescribed right-hand side is $F^{d}=R^{d}+M V^{-} . V$ is the velocity of the grain (it contains the translational degrees of freedom, and the rotational ones); $R$ is the resultant impulse on the grain due to interactions with other grains. The matrix $M$ contains both the mass (for the translational degrees of freedom) and the inertia (for the rotational degrees of freedom). A choice leading to get a constant, and diagonal, matrix $M$ consists in expressing the global coordinates of rotation vectors in the inertia eigenbasis of each grain, Figure 1. With such a choice, $R^{d}=R^{\text {ext }}+R^{\text {rot }}$, where $R^{\text {ext }}$ are the prescribed external forces and $R^{\text {rot }}$ are the fictitious forces defined as

$$
R^{\mathrm{rot}}=\left(\begin{array}{c}
0 \\
\omega \times I \omega
\end{array}\right)
$$

where $I$ is the moment of inertia and $\omega$ is the angular velocity. These fictitious forces are non linear with respect to the degrees of freedom. In the case of dense granular media, angular velocities are small enough to express $R^{\text {rot }}$ in an explicit way, by choosing the value obtained at $t_{i}$, as reported in [37]. This renders them explicitly known, and so, they are assembled into the right-hand side $R^{d}$, that contains also the prescribed impulse fields. The assembly of these equations (independent for each grain) for all the involved grains is formally written in the same way (1).

Contact interaction. Here we focus on simple unilateral contact which is naturally expressed as a complementary condition linking contact force to a gap. For dynamics, Moreau proved via a viability lemma [34], that we can use a velocity-impulse complementary law. The constitutive relation is summarized in the following formal equation:

$$
\mathcal{R}(v, r)=0
$$

$v$ is the velocity jump at the contact point between the two interacting grains, $r$ is the impulse at the same contact point. $\mathcal{R}$ is usually a non linear and multivalued relationship between the previous two dual quantities. For instance, 
for frictionless contact, it relates the normal components with the KKT complementary conditions $v_{n} \geq 0, r_{n} \geq 0$ and $v_{n} f_{n}=0$. Other models, such as extensible cables and frictional contact can be found in $[36,46]$. The assembly of the interaction-related quantities for all interactions is also written formally in the same way (2).

Both $v$ and $r$ are expressed in the local coordinate basis to the contacts between the interacting grains, Figure 1. Therefore, they are linked to the global kinematic and static quantities with a compatibility condition:

$$
v=H^{T} V \quad \text { and } \quad R=H r
$$

$H$ is the signed mapping between the global quantities related to the grains in their local basis $R_{i}$ with the local relative quantities related to the interactions in the local basis $R_{\alpha}$.

Reduced dynamics. Taking the dynamics (1) and the compatibility conditions (3) into account, the reduced dynamics involving material variables can be obtained:

$$
W r-v=-v^{d}
$$

where $W$ is the Delassus operator: $W=H^{T} M^{-1} H$, and $v^{d}=H^{T} M^{-1} F^{d}$.

To close the problem, one adds the constitutive relation (2), and the reference problem reads:

$$
\left\{\begin{array}{l}
W r-v=-v^{d} \\
\mathcal{R}(v, r)=0
\end{array}\right.
$$

This problem is classically solved within the NSCD (NonSmooth Contact Dynamics) method with a non-linear Gauss-Seidel (NLGS) solver [34, 24, 25].

Extension to deformable grains. Even if it will be not tested in the numerical results presented in the following, the case of modeling the grain behavior as an elastic deformable solid, with a finite element discretization, can be derived easily. This leads to even more large problems for which a domain decomposition method has also potentials for larger gains. Such a modeling is suited in particular for granular materials where deformation and eventually fracture of grains is under concern.

Now, the kinematic global unknowns $V$ are the whole set of translational degrees of freedom of the nodes, $K$ is the classical finite element stiffness matrix of the grain and $M$ is the mass matrix of the grain. Some care must nevertheless be taken with this mass matrix to get a discretized well-posed problem, see for instance $[26]$.

A co-rotational formulation $[1,2]$ has several advantages: if the rotations are finite, but the deformation is small, expressing the degrees of freedom in the inertia eigenbasis of the grain allows to consider constant operators $M$ and $K$. In such a case, as previously, the Coriolis and centrifugal effects are explicitly written, and are part of the given right-hand side of generalized forces $F_{d}$.

A two-scale description consists in setting $V=R_{s} V_{s}+V_{e}$ where $V_{s}$ is the previously described small-sized vector of the global rigid body movement of the grain, at its center of mass. $R_{s}$ is the extension of this movement to all the nodal degrees of freedom of the discretized body (in its inertial eigenbasis). $V_{e}$ is an additional movement mainly containing the elastic deformation of the 
grain (in its inertial eigenbasis as well), to be precised in the following. The non smooth dynamics of the grain therefore reads:

$$
M V+R_{\text {int }}-R=F^{d}
$$

with the internal impulse

$$
R_{\mathrm{int}}=\int_{t_{i}}^{t_{i+1}} K U d t
$$

where $U$ is the nodal vector of the displacement in the inertial eigenbasis). Using the test functions $V^{\star}=R_{s} V_{s}^{\star}+V_{e}^{\star}$, the dynamics leads to

$$
\begin{array}{r}
M V_{e}+R_{\mathrm{int}}+M R_{s} V_{s}-R=F^{d} \\
\bar{M} V_{s}+R_{s}^{T} R_{\mathrm{int}}+R_{s}^{T} M V_{e}-\bar{R}=\bar{F}^{d}
\end{array}
$$

where $\bar{M}=R_{s}^{T} M R_{s}, \bar{R}=R_{s}^{T} R, \bar{F}^{d}=R_{s}^{T} F^{d}$.

With a constant stiffness matrix, one coupling term is

$$
R_{s}^{T} R_{\mathrm{int}}=R_{s}^{T} K \int_{t_{i}}^{t_{i+1}} U d t=0
$$

since $K R_{s}=0$, and, to ensure the uniqueness of the two-scale description, we choose as an orthogonality condition between the two kinematic spaces: $R_{s}^{T} M V_{e}=0$ that cancels the second coupling term in (9), which is therefore identical to the rigid model (1). Once $V_{s}$ is obtained, the "deformable" component $V_{e}$ then arises from (8):

$$
M V_{e}+R_{\mathrm{int}}-R=F^{d}-M R_{s} V_{s}
$$

The last step is to link the displacement update $U$ to the velocity $V$. A possibility is to obtain it from two sources: $U=U_{s}+U_{e}$, where $U_{s}$ is a rigid body rotational finite movement (useful to update the finite rotation of the inertial eigenbasis), and $U_{e}$ corresponds to the complementary part. $U_{s}$ can be obtained with the Hughes-Winget scheme [22] or the Rodrigues formula, while $U_{e}$ can be obtained with a $\theta$-method as a time integration scheme. Neglecting the residual $K U_{s}^{-}$in algebraic developments, one gets the internal impulses as

$$
R_{\mathrm{int}}=h K U_{e}^{-}+h \theta\left[h(1-\theta) K V_{e}^{-}+h \theta K V_{e}\right]
$$

where the time step is $h=t_{i+1}-t_{i}$. The corresponding dynamics therefore reads:

$$
\tilde{M} V_{e}-R=\tilde{F}^{d}-M R_{s} V_{s}
$$

where $\tilde{F}^{d}$ is a given right-hand side, and $\tilde{M}=M+h^{2} \theta^{2} K$. One can check that, with the coupling term $M R_{s} V_{s}$, the dynamics (10) gives a solution that satisfy to the orthogonality condition. Indeed, by pre-multiplying (8) with $R_{s}^{T}$, one can easily check that it leads to $R_{s}^{T} M V_{e}=0$.

Finally, the reduced dynamics can also be drawn for this model case, as previously with a new definition of matrix $H$ which is now correlated to the whole set of degrees of freedom, but with the same expression as in (3):

$$
v=H^{T}\left(R_{s} V_{s}+V_{e}\right) \quad \text { and } \quad R=H r, \quad \bar{R}=R_{s}^{T} H r
$$


which leads to

$$
\left\{\begin{array}{l}
\tilde{W} r-v=-\tilde{v}^{d} \\
\mathcal{R}(v, r)=0
\end{array}\right.
$$

with, in fine, $\tilde{W}=H^{T} \tilde{M}^{-1} H$ and $\tilde{v}^{d}=H^{T} \tilde{M}^{-1} \tilde{F}^{d}$. Therefore, the problem characteristics are very close to the ones obtained from the case of rigid grains and algorithmic choices in a domain decomposition approach should be valid for both modelings.

\subsection{Domain partitioning}

The domain has to be split into subdomains in order to use parallel computing. This decomposition is performed as frequently as needed to take into account the migration of grains from one subdomain to another. Such a strategy may be implemented with minimal computational efforts using sophisticated routines out of the purpose of this paper. Since the nonsmoothness may occur in interactions between grains, we choose to distribute interactions among subdomains as in [4] (we proceed by distributing the middle points between the centers of mass of interacting grains, according to their coordinates, using an arbitrary regular underlying grid, Figure 2 and Figure 3). Indeed, with such a choice, the "boundary" grains are duplicated in the two subdomains. If a grain indexed with $i$ is connected with $m^{i}$ subdomains, $m^{i}$ is called its multiplicity number. For consistency for the rigid model of the grains, the masses and moments of inertia are distributed among the neighboring subdomains according to their multiplicity number. More precisely the distribution of masses and inertia is an algebraic partitioning and not a geometrical partitioning. It is more meaningful to speak about a duplication of the boundary grains than a splitting of them. For the elastic deformable model of the grains, this splitting can be performed with a classical mesh decomposer. The interface between two subdomains is defined as the set of these grains, that joins the subdomains. The nonsmoothness is therefore localized only within the subdomains. This modeling choice is identical to [10] and somehow the dual of that proposed in [28], where nonlinearities (contact on crack lips) are isolated at the interfaces. Note that a direct algebraic partitioning of the reference problem can also be chosen, leading to a dual partitioning and a different algorithm [21]. Some advantages and disadvantages have been mentioned in the introduction but such a topic has to be investigated more deeply in forthcoming works.

\subsection{FETI-like formulation and NSCDD algorithm}

In each subdomain $E$, the problem is identical to the global one (with the subscript $E$ ), provided that a term arising from the inter-grain interface is added. It is therefore described in the following only for the rigid model of the grains. It can be built from the interconnecting condition (on the velocities of boundary grains) that has been added to "glue" neighboring subdomains, where $A_{\Gamma E}$ is a signed boolean matrix with a finite rotation, to map the grain velocities $V_{E}$ to the global coordinate basis into which the interconnectivity is expressed:

$$
\sum_{E=1}^{n_{\text {sd }}} A_{\Gamma E} V_{E}=0
$$



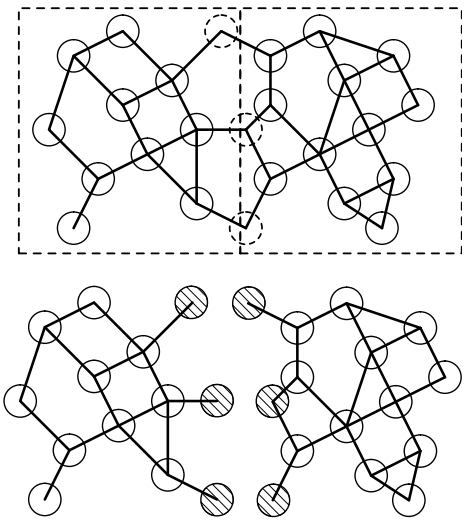

Figure 2: Geometrical partitioning of the discrete domain and duplication of the interface grains.
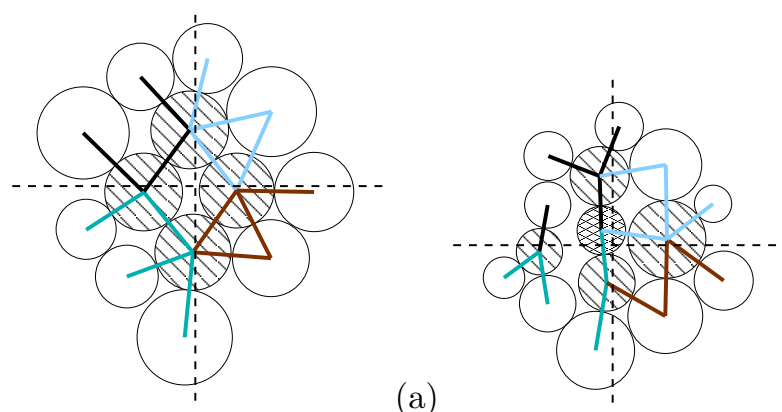

(b)

Figure 3: Illustrations of the proposed domain partitioning technique: four grains having a multiplicity of 2 (a) (cf. Figure 6(b)), four grains having a multiplicity of 2 and one having a multiplicity of 3 (cf. Figure 10(b)). Contacts are colored according to the subdomain they belong to. The grains having a multiplicity of 2 are hatched, the grain having a multiplicity of 3 is crossedhatched. 
$\Gamma$ denotes the global interface between all the neighboring grains. Formally the previous summation is performed on all the subdomains (number equal to $n_{\mathrm{sd}}$ ), even if, for a given interface grain the only neighboring subdomains have to be considered. Then we obtain a FETI-like formulation [18] for the reference problem using a multiplier field $F_{\Gamma}$ and the notations $\hat{A}_{\Gamma E}^{T}=H_{E}^{T} M_{E}^{-1} A_{\Gamma E}^{T}$ :

$$
\begin{aligned}
& \left.\begin{array}{l}
W_{E} r_{E}-v_{E}-\hat{A}_{\Gamma E}^{T} F_{\Gamma}=-v_{E}^{d} \\
\mathcal{R}\left(v_{E}, r_{E}\right)=0
\end{array}\right\} E=1, \ldots, n_{\mathrm{sd}} \\
& \sum_{E=1}^{n_{\text {sd }}} A_{\Gamma E} V_{E}=0
\end{aligned}
$$

The reduced problem on $\left(r_{E}, v_{E}, F_{\Gamma}\right)$, with the notations $\hat{f}=\sum_{E} A_{\Gamma E} M_{E}^{-1} F_{E}^{d}$ and $X=\sum_{E} A_{\Gamma E} M_{E}^{-1} A_{\Gamma E}^{T}$, reads:

$$
\begin{aligned}
& \left.\begin{array}{l}
W_{E} r_{E}-v_{E}-\hat{A}_{\Gamma E}^{T} F_{\Gamma}=-v_{E}^{d} \\
\mathcal{R}\left(v_{E}, r_{E}\right)=0
\end{array}\right\} E=1, \ldots, n_{\mathrm{sd}} \\
& X F_{\Gamma}-\sum_{E=1}^{n_{\text {sd }}} \hat{A}_{\Gamma E} r_{E}=\hat{f}
\end{aligned}
$$

As for many domain decomposition approaches, the goal is to be able to localize the same typical problem that is under consideration on each subdomain independently, while designing a suited coupling recovery algorithm between subdomains, i.e. on the interface.

Here, the algorithmic formulation described in Algorithm 1 has been implemented into the LMGC90 platform [16] for time-evolution problems $(N$ is the number of time steps). At each new time step of the incremental solving procedure, the mapping $H$ and the contact graph have to be reevaluated within a contact detection phase. Eventually, the domain could also be repartitioned according to the new contact graph. For each time step, the iterative resolution proceeds with several stages. First, the interface impulses obtained at the previous iteration are disassembled into $F_{E}=-A_{\Gamma E}^{T} F_{\Gamma}$ that is considered as given additional external impulses in the subdomain $E$, and added to the prescribed values $F_{E}^{d}$. At each iteration, the solver is itself a predictor-corrector scheme, for which a "free" grain velocity is first computed as $\bar{V}_{E}^{d}=M_{E}^{-1}\left(F_{E}^{d}+F_{E}\right)$. At the interaction level, one then computes $\bar{v}_{E}^{d}=H_{E}^{T} \bar{V}_{E}^{d}$. The correction phase is composed with an incomplete solving procedure of the nonsmooth dynamics problem on each subdomain, with $n_{\text {GS }}$ prescribed iterations of non-linear GaussSeidel algorithm. This provides the local impulses $\bar{r}_{E}$ (satisfying the reduced dynamics, even if the solve is incomplete). The resultant impulse per grain is $\bar{R}_{E}=H_{E} \bar{r}_{E}$, and the correction reads: $\bar{V}_{E}=\bar{V}_{E}^{d}+M_{E}^{-1} \bar{R}_{E}$.

Up to this point, it is interesting to note that the interface problem in (15) can be stated in a correction form, using $\Delta F_{\Gamma}=\bar{F}_{\Gamma}-F_{\Gamma}$ : noting that $\bar{r}_{E}$ satisfies to the grain dynamics, this interface problem can be restated as

$$
X \Delta F_{\Gamma}=\hat{f}+\sum_{E=1}^{n_{\mathrm{sd}}} \hat{A}_{\Gamma E} \bar{r}_{E}-X F_{\Gamma}=\sum_{E=1}^{n_{\mathrm{sd}}} A_{\Gamma E} \bar{V}_{E}
$$

the last term being the residual on the interface, i.e. the velocity jump.

At each time step, inner iterations are stopped when the classical NLGS convergence criterion (on the subdomains, [12]) and the gluing criterion over the interface [23] are verified. 


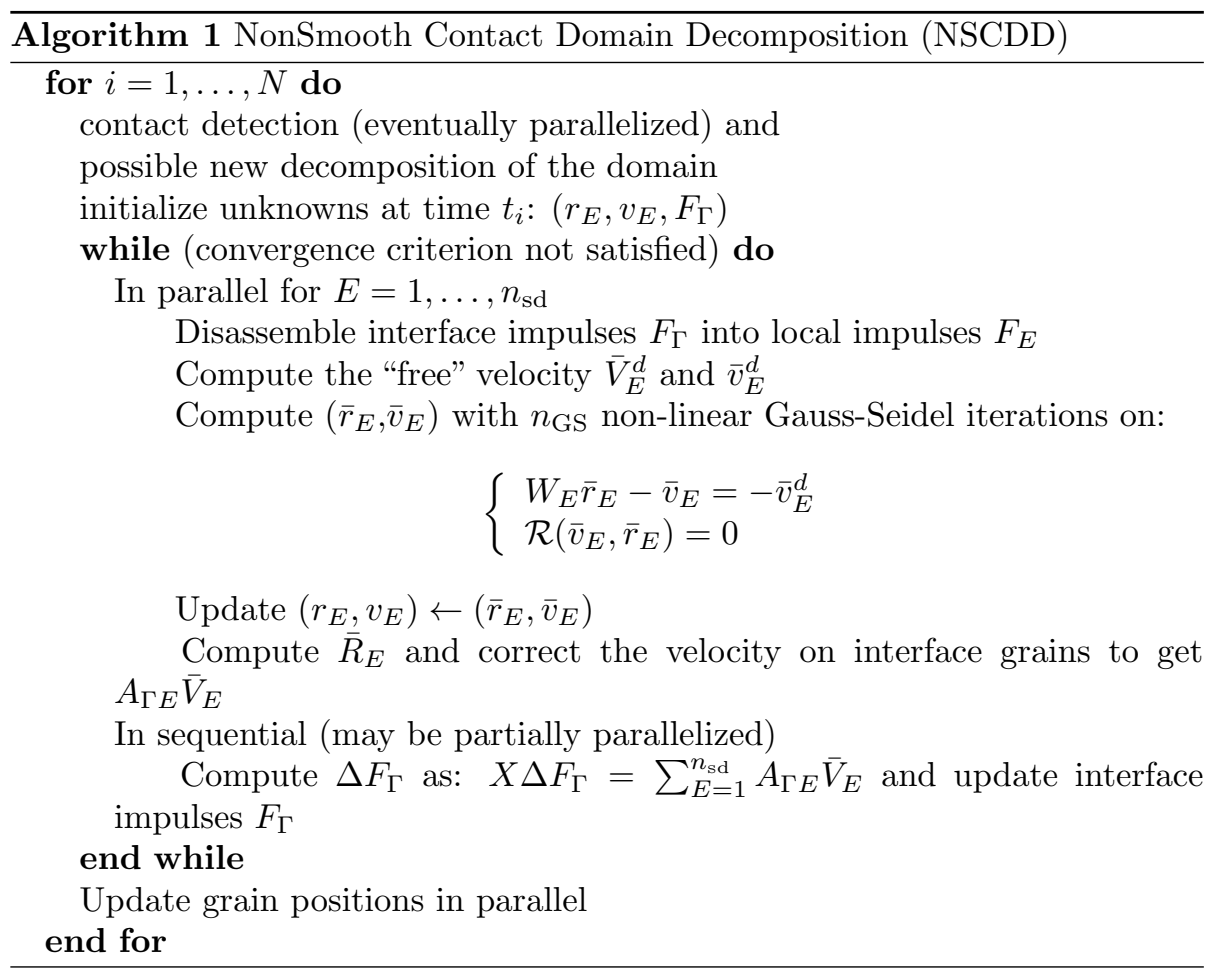

\section{Interface topics}

For discrete systems, several specificities of the interface treatment are detailed in this section, in particular the structure of the interface operator $X$.

\subsection{Structure of the interface}

For discrete systems, the global interface $\Gamma$ is constituted of grains supporting contacts in more than one subdomain. The number of subdomains a grain $i$ is connected to is called its multiplicity $m^{i}$. As for classical domain decomposition methods [43] one gets $m_{E}=1+A_{\Gamma E}^{T} A_{\Gamma E}$ as the diagonal matrix whose entries for each grain $i$ kinematic dof is $m^{i}$. Depending on their multiplicity, the grains are called "internal grains" if $m^{i}=1$ (otherwise, they will be called "interface grains"), "face grains" if $m^{i}=2$ and "corner grains" if $m^{i}>2$. Contrary to the continuous media case where face, edge and corner nodes can be distinguished in $3 \mathrm{D}$, the discrete systems we are considering here do not differentiate edge and corner topology.

Corner grains are split in $m^{i}$ parts and links are stated as gluing conditions between these parts (the impulses in such gluing conditions are stored in $F_{\Gamma}$ ). Sufficient gluing conditions should be stated for each interface grains (face or corner) to ensure to recover the reference problem solution. Several options are:

- Discard the treatment of corner grains. This option can be used for several DDM for the continuous media case when interface fields are defined at the finite element level on edges of elements rather than at nodes $[17,11]$ since the measure of corner nodes is zero. For a discrete model as considered 
herein, this is not an available option since then, continuity at these grains won't be taken into account.

- Consider as many gluing conditions as the mutiplicity of the considered corner grain: $n_{l}^{i}=m^{i}$. In this case, there is a small overconstrained gluing condition (only $m^{i}-1$ links are sufficient to glue $m^{i}$ parts).

- Consider an even larger number of gluing condition, similarly to redundant corner conditions in FETI methods [43]. The maximal number of conditions that can be established between $m^{i}$ parts is $\frac{1}{2} m^{i}\left(m^{i}-1\right)$.

In order to avoid singularity of the interface operator $X$, and to allow several solving procedures for the interface problem, we choose to prescribe the necessary and sufficient number of gluing conditions on corner grains, i.e. $m^{i}-1$ conditions only.

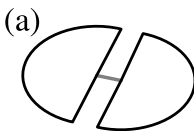

(d)

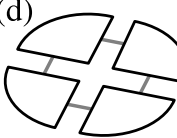

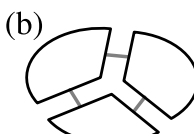

(e)

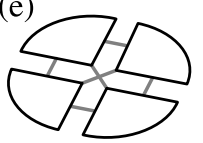

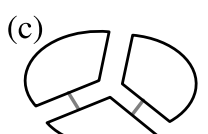

(f)

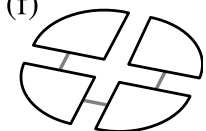

Figure 4: Gluing conditions between interface grains. (a) $m^{i}=2$ and $n_{l}^{i}=$ $m^{i}-1=\frac{1}{2} m^{i}\left(m^{i}-1\right)=1$; (b) $m^{i}=3$ and $n_{l}^{i}=m^{i}=\frac{1}{2} m^{i}\left(m^{i}-1\right)=3$; (c) $m^{i}=3$ and $n_{l}^{i}=m^{i}-1$; (d) $m^{i}=4$ and $n_{l}^{i}=m^{i}$; (e) $m^{i}=4$ and $n_{l}^{i}=\frac{1}{2} m^{i}\left(m^{i}-1\right)=6$; (f) $m^{i}=4$ and $n_{l}^{i}=m^{i}-1$. (For convenience the grains have been split even if they are not geometrically cut.)

\subsection{Analysis of the interface operator $X$}

Internal grains $\left(m^{i}=1\right)$ indeed have no contribution to $X$. When only face grains exist $\left(m^{i}=2\right), X$ has been proved to be diagonal per block, i.e. each grain is decoupled with the other ones [4] and each block is at most of size $(b, b)$ where $b$ is the number of kinematic or static components for each grain: $b=3(D-1)$ for rigid grains where $D=2,3$ is the dimension of the considered physical space. In a dynamic framework "sthenic" may be preferred to "static" in reference to a description of dual variables to the kinematics. This is a specific issue of the dynamics of rigid grains: the interface problem does not condense information from the inner part of subdomains. Moreover, each block may itself be diagonal for special cases (circular or spherical grains). In these cases, solving the interface problem is trivial. Corner grains do indeed modify the structure of this operator. It is still block-diagonal, but a full block occurs on links related to each corner grain $i$ with a size $\left(n_{l}^{i} b, n_{l}^{i} b\right)$.

Consider the contribution of corner grain $i$ from the various subdomains that share this grain; this corresponds to a block in matrix $X$ denoted as:

$$
X^{i}=\sum_{E=1}^{n_{\mathrm{sd}}} A_{\Gamma E}^{i}\left(M_{E}^{i}\right)^{-1}\left(A_{\Gamma E}^{i}\right)^{T}
$$


There are no gluing impulse $F_{\Gamma}$ between different corner grains, so blocks $X^{i}$ are decoupled to each other. $M_{E}^{i}$ is the mass matrix for the part of grain $i$ located in subdomain $E$. Since they are always symmetric, positive definite, and for sake of simplicity, they are omitted in the following developments (they are all considered as identity matrices, which does not change the structure of the interface operator), moreover, all the following matrix entries will correspond to a block of size $(b, b)$ corresponding to a whole set of kinematic or static (or sthenic) components. With an arbitrary sign convention in $A_{\Gamma E}^{i}$, and for $n_{l}^{i}=m^{i}-1$ gluing conditions, which is an open cyclic connectivity graph, the block $X^{i}$ is a permutation of the following pattern:

$$
X^{i}=\left[\begin{array}{rrrr}
2 & -1 & & \\
-1 & \ddots & \ddots & \\
& \ddots & 2 & -1 \\
& & -1 & 2
\end{array}\right]
$$

which is clearly invertible. Adding an additional gluing condition will close a sub-cycle in the connectivity, and therefore produces a sub-block in $X^{i}$ with the pattern

$$
\left[\begin{array}{rrrr|r}
2 & -1 & & & 1 \\
-1 & \ddots & \ddots & & \\
& \ddots & 2 & -1 & \\
& & -1 & 2 & 1 \\
\hline 1 & & & 1 & 2
\end{array}\right]
$$

which turns out to be singular (last row is the sum of all the previous ones). Since this is a minor of $X^{i}$, this last matrix will be singular as soon as $n_{l}^{i}>m^{i}-1$.

The convergence study of the NLGS algorithm is quite complicated and will not be discussed herein. Nevertheless, simulations using corner grains will exemplify the impact of those grains on the physical properties of the granular system are discussed in the following.

\section{A mechanical study as a validity test}

The issue of this section is to test the robustness of the DDM approach with respect with various "well-known" aspects of the mechanical behavior of model granular media. To do so, at a first hand, we describe the numerical samples, and compare the macroscopic response of sheared granular packings for different decompositions. The microstructure (i.e. the spatial organization of the particles and their contacts) is analyzed at a second hand as a function of the number of subdomains.

\subsection{Simulation of a biaxial test}

A dense packing composed of 12000 disks is first set up by means of a layerby-layer deposition model based on simple geometrical rules [49]. The particles are deposited sequentially on a substrate. Each new particle is placed at the lowest possible position on the free surface as a function of its diameter. This 
procedure leads to a random close packing in which each particle is supported by two underlying particles and supports one or two other particles. To avoid long range ordering a small polydispersity in size is used.

(a) $\sigma_{0}$

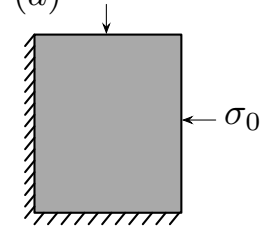

(b) $v_{y}$

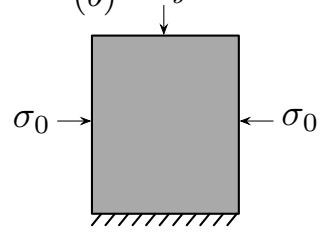

Figure 5: Boundary conditions for (a) isotropic and (b) biaxial compactions.

Following this geometrical process, the packing is compacted by isotropic compression inside a rectangular frame of dimensions $l_{0} \times h_{0}$ in which the left and bottom walls are fixed, and the right and top walls are subjected to a compressive stress $\sigma_{0}$. The gravity $g$ and friction coefficients $\mu$ between particles and with the walls are set to zero during the compression in order to avoid force gradients and obtain isotropic dense packings, see Figure 5(a). The isotropic samples are then subjected to a vertical compression by downward displacement of the top wall with a constant velocity $v_{y}$ for a constant confining stress $\sigma_{0}$ acting on the lateral wall, see Figure 5(b). The friction coefficient $\mu$ between particles is set to 0.35 and to zero with the walls. Since we are interested in quasistatic behavior, the shear rate should be such that the kinetic energy supplied by shearing is negligible compared to the static pressure. This can be formulated in terms of an inertia parameter I defined as [20]:

$$
I= \begin{cases}\dot{\varepsilon} \sqrt{\frac{m}{p}} & \text { in } 2 \mathrm{D}, \\ \dot{\varepsilon} \sqrt{\frac{m}{p d}} & \text { in } 3 \mathrm{D},\end{cases}
$$

where $\dot{\varepsilon}=\dot{y} / y$ is the strain rate, $m$ is the mean particle mass, $d$ the mean diameter and $p$ is the mean pressure (defined as the force per unit width for the $2 \mathrm{D}$ case). The quasistatic limit is characterized by the condition $I \ll 1$; in the proposed simulations, $I$ is below $10^{-4}$.

This simulation is repeated 5 times, with various numbers of subdomains ranging from 0 (corresponding to the reference simulation) up to 4 (they are tagged in the following with $S 0$ up to $S 4$ ). Figure 6(a) depicts the five decompositions we choose at the initial state. Figure $6(\mathrm{~b})$ is a zoom of the case $S 4$ to illustrate the particle arrangement.

\subsection{Macroscopic behavior}

In this section, we consider the stress-strain and volume-change behavior according to the domain decomposition. We therefore need to evaluate the stress tensor and solid fraction during deformation from the simulation data at microscopic scale. 


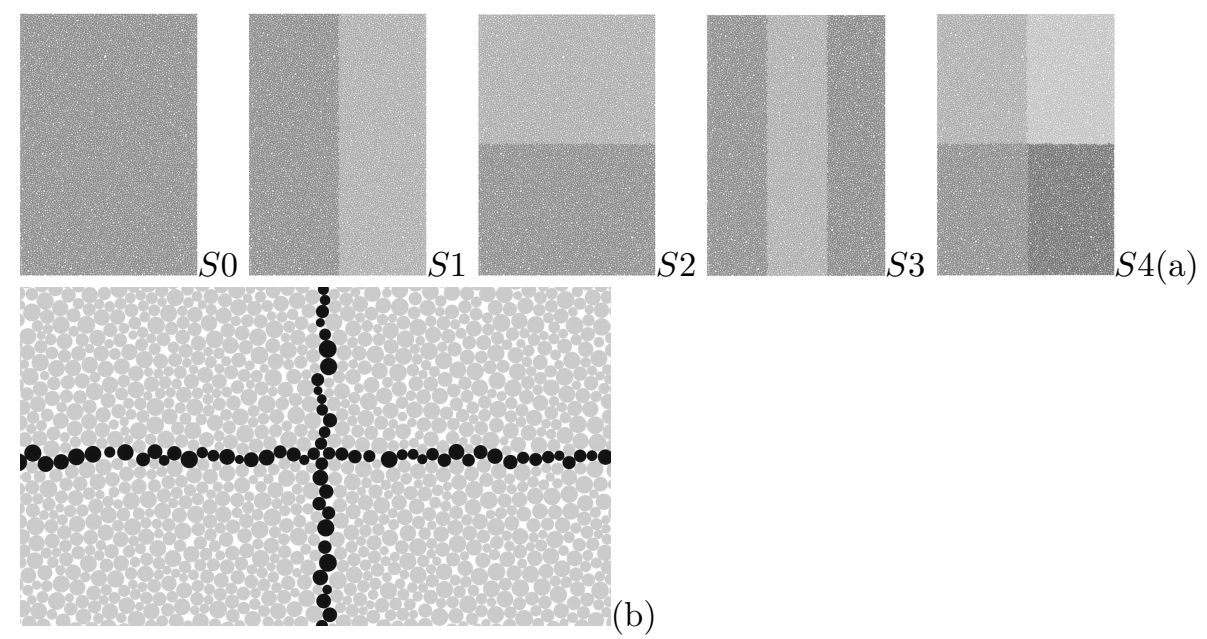

Figure 6: Examples of decomposition at the initial state (a) and zoom at the intersection of the four subdomains of case $S 4$ (b). The multiplicity is (b): 1 for a gray particle and 2 for a black particle.

\subsubsection{Definition of some macroscopic parameters}

In granular media, the expression of the stress tensor $\sigma$ in the volume $V$ is an arithmetic average involving the branch vectors $\ell^{\alpha}$ (joining the centers of the two neighbouring particles) and the contact force vectors $f^{\alpha}$ at contact $\alpha$. It is given with [33, 47]:

$$
\sigma=\frac{1}{V} \sum_{\alpha \in V} f^{\alpha}\left(\ell^{\alpha}\right)^{T}
$$

Under biaxial conditions with vertical compression, we have $\sigma_{1} \geq \sigma_{2}$, where the $\sigma_{k}$ are the stress principal values. The mean stress is $p=\left(\sigma_{1}+\sigma_{2}\right) / 2$, and the stress deviator is $q=\left(\sigma_{1}-\sigma_{2}\right) / 2$. According to the Mohr-Coulomb model, the shear strength of dry granular materials can be linked to the internal angle of friction $\varphi$ as follows [31]:

$$
\sin \varphi=\frac{q}{p}=\frac{\sigma_{1}-\sigma_{2}}{\sigma_{1}+\sigma_{2}}
$$

The vertical macroscopic strain $\varepsilon_{1}$ is the cumulative value defined as:

$$
\varepsilon_{1}=\int_{h_{0}}^{h} \frac{d h^{\prime}}{h^{\prime}}=\ln \left(1+\frac{\Delta h}{h_{0}}\right)
$$

where $h_{0}$ is the initial height and $\Delta h=h_{0}-h$ is the total downward displacement.

\subsubsection{Shear strength and solid fraction}

Figure 7 depicts the internal angle of friction $\sin \varphi$ as a function of the shear strain $\varepsilon_{1}$ for all the decompositions. The jump observed at $\varepsilon_{1}=0$ reflects both the rigidity of the particles and the large initial solid fraction of the samples. In all cases, the shear stress passes by a peak $(q / p)^{\text {peak }} \sim 0.38$ before relaxing 


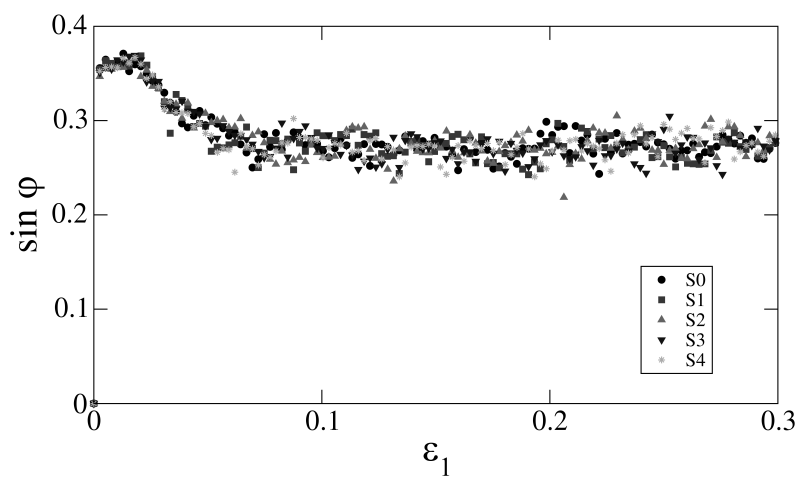

Figure 7: Internal angle of friction $\sin \varphi$ as a function of the vertical deformation $\varepsilon_{1}$.

to a stress plateau $(q / p)^{*} \sim 0.28$ corresponding to the so-called "residual state" in soil mechanics [31]. We see that, up to the fluctuations, all curves join nicely on the same curve.

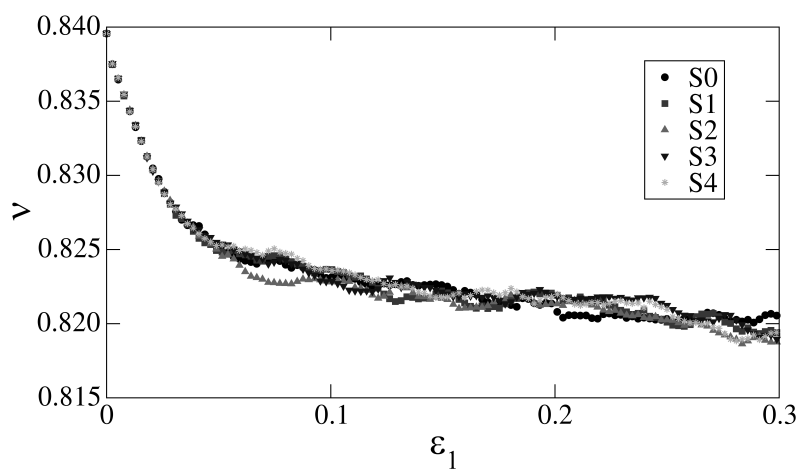

Figure 8: Solid fraction $\nu$ as a function of the vertical deformation $\varepsilon_{1}$.

Figure 8 shows the variation of the solid fraction $\nu=V_{p} / V$ as a function of $\varepsilon_{1}$ for all the decompositions, where $V_{p}$ is the volume (area in 2D) occupied by the particles. The solid fraction decreases first from $\nu_{0} \simeq 0.84$ to 0.825 . It is remarkable that, during this phase the solid fraction is rigorously identical for all the samples. At larger strains, the solid fraction decreases much more slowly and, up to the fluctuations, saturates on the same curve. Indeed, at larger strains, dilation is localized within shear bands appearing and vanishing throughout the system underlying the saturation of $\nu$. This is well illustrated in Figure 9 where two maps of the particle velocities are shown at $\varepsilon_{1}=0.2$ for $S 0$ and $S 3$ cases. We see clearly that the topology of the shear band are slightly different even if, on average, the solid fraction is identical. In fact, localization phenomena leads to multiple possible physical solutions, and it has been already been exemplified that the formation of the shear band depends on the details of the numerical parameter of the simulations (time step, solver, number of iterations...) [41].

In this section we have shown that the macroscopic response of a sheared 

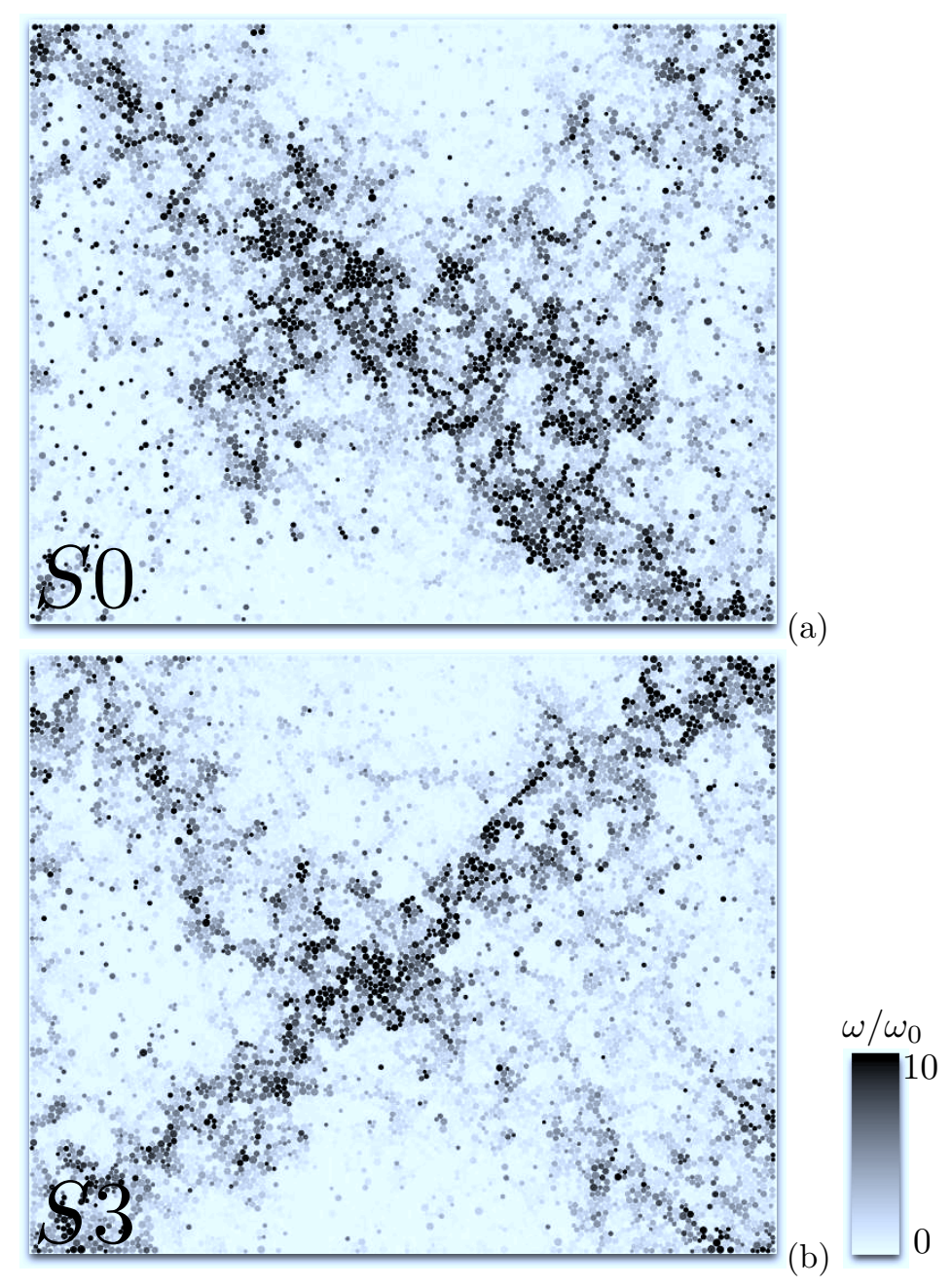

Figure 9: Maps of the normalized angular velocities of the particle for $S 0$ (a) and $S 3$ (b) cases, at $\varepsilon_{1}=0.2$. 
granular material is independent of the chosen number of subdomains. Nevertheless, a granular material is a typical example of multi-scale material: the macroscopic behavior results from the average properties of a collection of interacting particles through the contact network. This is clearly illustrated in the case of elongated particles where the residual shear strength increases linearly with elongation [7], whereas for angular or non-convex particle shapes, the residual shear strength increases first and saturate as the level of angularity or non-convexity of the particles is increased [6, 45]. An other surprising effect is that the residual shear strength is independent of the polydispersity [50]. This wide variety of behaviors finds its origins at the scale of the particle and contact properties. Thus, we also need to test the robustness of the domain decomposition solver in terms of the granular microstructure.

\subsection{Micromechanical analysis}

The granular microstructure (granular texture), i.e. the spatial organization of the particles and their contacts, is basically controlled by steric exclusions between the particles and force balance conditions [48]. The strong inhomogeneity of contact forces is a well known feature of granular media. Figure 10 shows the contact forces for $S 2$ and $S 4$ cases at the residual state. For the same level of strain, the force-carrying backbones are different, even if the global inhomogeneity seems to be preserved. Of course, this is due to the fact that the resolution of the contact forces is performed domain by domain, and to the plurality of local solutions for frictional granular media. Nevertheless, this anisotropic structure, generally at the origin of the shear strength of granular media, can be described more rigorously in terms of various statistical descriptors pertaining to the forcebearing network of particles. In the following, we consider two aspects of the microstructure: (i) the angular average of the contact and force orientations, and (ii) the normal force distributions.

\subsubsection{Contact and force anisotropy}

A common approach is to consider the probability distribution of the contact normals $n$, which is usually nonuniform. As shown in Figure 1, for the 2D case the unit vector $n$ is described by a single angle $\theta$. The probability density function $P(\theta)$ of contact normals provides detailed statistical information about the texture. In the same way, expressing the force vector in the local contact frame $(n, t)$, where $t$ is an orthonormal unit vector oriented along the tangential direction, we can compute the angular distributions $\left\langle f_{n}\right\rangle(\theta)$ and $\left\langle f_{t}\right\rangle(\theta)$ of normal and tangential forces, respectively. The above three functions describe the general state of the packing. Under shearing, the packing organizes itself into a state where these functions are well approximated with their lowest-order Fourier expansion $[44,27,39,7]$ :

$$
\left\{\begin{array}{l}
P_{\theta}(\theta)=\frac{1}{2 \pi}\left\{1+a_{c} \cos 2\left(\theta-\theta_{c}\right)\right\} \\
\left\langle f_{n}\right\rangle(\theta)=\langle f\rangle\left\{1+a_{f n} \cos 2\left(\theta-\theta_{f n}\right)\right\} \\
\left\langle f_{t}\right\rangle(\theta)=\langle f\rangle a_{f t} \sin 2\left(\theta-\theta_{f t}\right)
\end{array}\right.
$$

where $a_{c}, a_{f n}$, and $a_{f t}$ are the anisotropy parameters and $\theta_{c}, \theta_{f n}$, and $\theta_{f t}$ represent the corresponding privileged directions. In a sheared state the privileged directions tend to follow the principal stress direction (i.e. $\theta_{c}=\theta_{f n}=\theta_{f t}=\theta_{\sigma}$ ). 

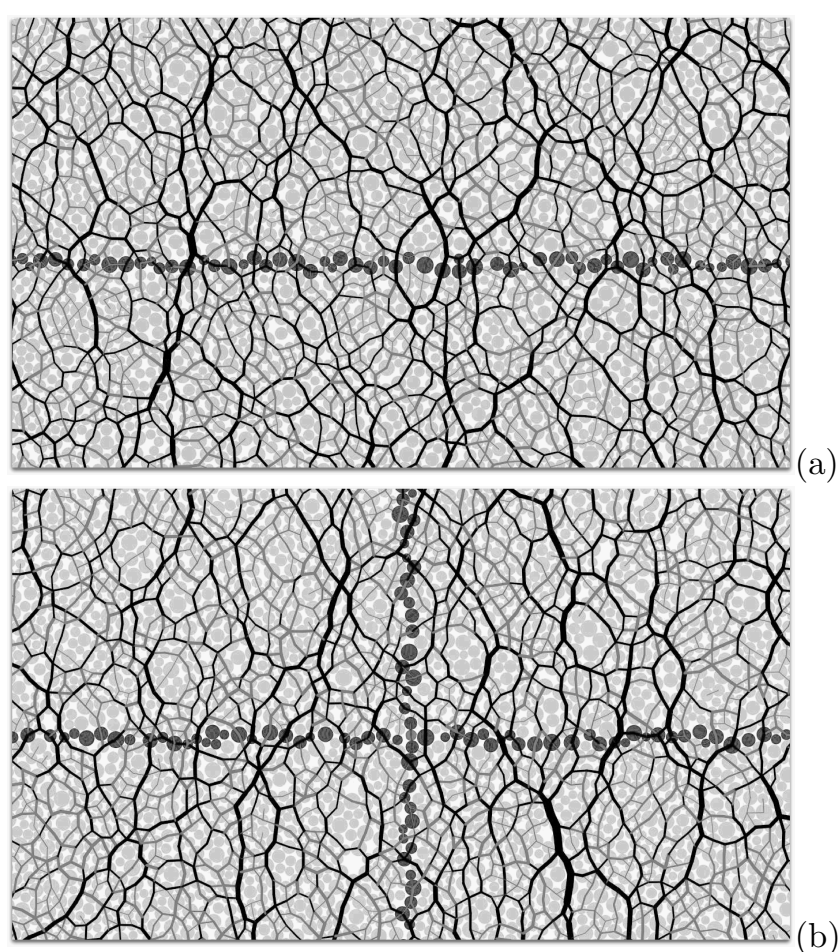

a)

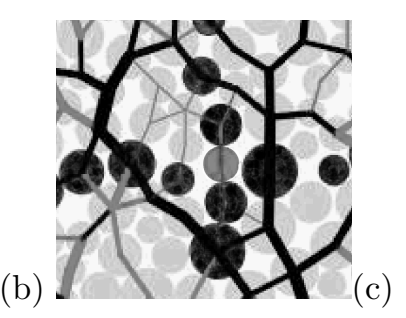

Figure 10: Maps of force chains in a portion of $S 2$ (a) and $S 4$ (b) samples, at $\varepsilon_{1}=0.2 ;$ (c) is a zoom on map at the subdomains intersection (b). Line thickness is proportional to the normal force. Strong forces $\left(f_{n}>\left\langle f_{n}\right\rangle\right)$ are in black and weak forces $\left(f_{n}<\left\langle f_{n}\right\rangle\right)$ in gray (see text). The multiplicity is: 1 for a light gray particle, 2 for a medium gray particle and 3 for a black particle. 
In practice the values of all anisotropy parameters can be computed from generalized fabric and force tensors presented in $[40,7]$.
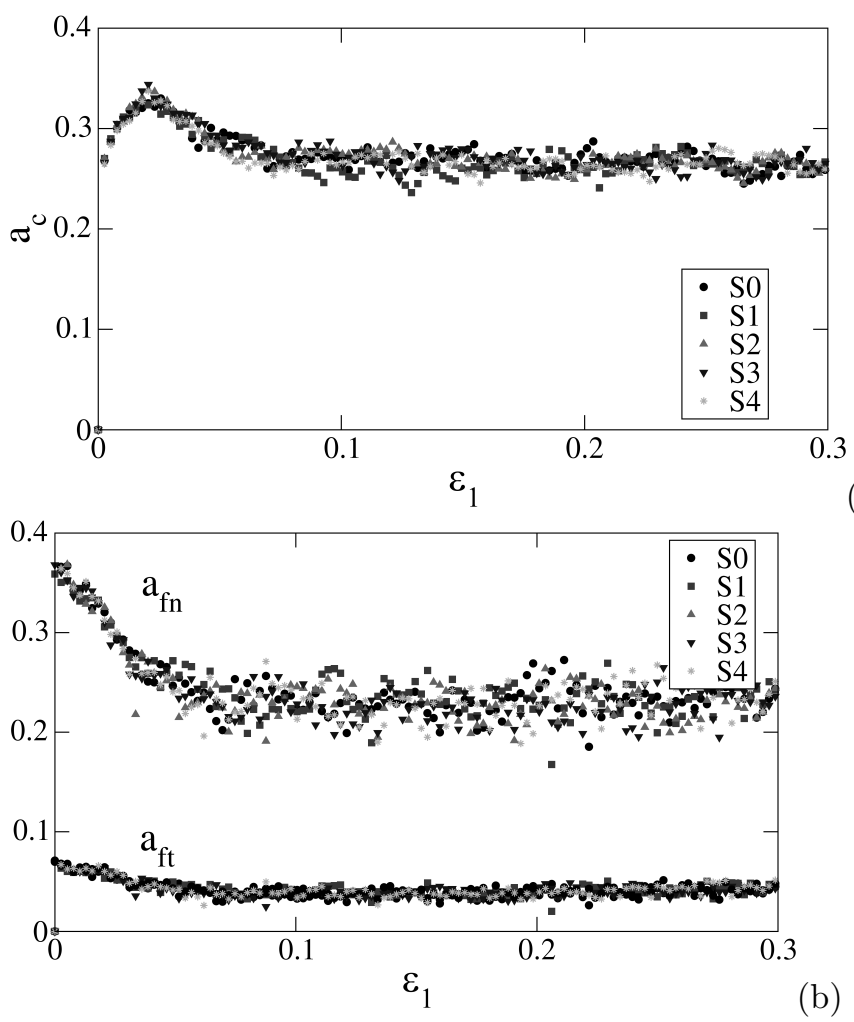

(a)

Figure 11: Contact anisotropy (a) and normal and tangential force anisotropies (b) as functions of the vertical deformation $\varepsilon_{1}$ for all the tested decompositions.

Figure 11 shows the variations of all these anisotropy parameters as functions of $\varepsilon_{1}$ for all the domain decompositions. Up to fluctuations, all the curves join also nicely on the same curve. We see that $a_{c}$ follows the same trend as the shear strength, increases first to a maximum value equal to $\simeq 0.35$ and then declines to a plateau at $\simeq 0.26$. In contrast, $a_{f n}$ decreases from $\simeq 0.38$ to a constant value $\simeq 0.24$, whereas $a_{f t}$ remains nearly constant with the strain.

These anisotropy parameters are very relevant to the analysis of the granular microstructure because they can bring to light the geometrical and mechanical origins of the shear strength. Indeed, it can be shown that the general expression of the stress tensor (21) leads to the following "stress-force-fabric" relationship (a term coined for the first time by Rothenburg and Bathurst in [44]):

$$
\sin \varphi \simeq \frac{1}{2}\left(a_{c}+a_{f n}+a_{f t}\right)
$$

where the cross products between the anisotropy parameters have been neglected. It is very important to test the validity of relation (24) in the context of numerical simulation by DDM because this equation reveals an explicit link between microscopic and macroscopic scales in granular media. We plot in Figure 12 the variation of the shear strength together with the harmonic fit of 
equation (24). We see that data are in quantitative good agreement with this harmonic approximation which is not affected by the number of subdomains.

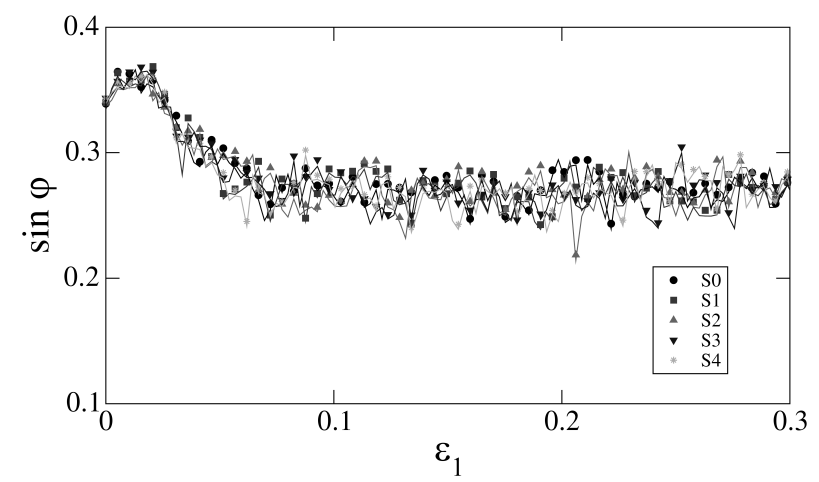

Figure 12: Friction angle $\sin \varphi$ (symbol) together with the harmonic approximation (line) as functions of the vertical strain $\varepsilon_{1}$ for all the tested decompositions.

\subsubsection{Force distribution}

Force transmission has been investigated by experiments and numerical simulations for disks, elongated, polygonal and non-convex particles in 2D as well as for spherical, cylindrical and polyhedral particles in 3D [38, 13, 35, 5, 42, 8, 9]. The probability density function (pdf) of normal forces is characterized by two features that are specific to granular media: (i) the pdf is roughly a decreasing exponential function for forces above the mean value, (ii) in the range of weak forces below the mean value, the pdf does not decline to zero with the force. The relative scattering of data reported by different authors for weak forces shows the sensitivity of the pdf within this range to the microstructure details.

The probability density function (pdf) of normal forces normalized by the mean normal force $\left\langle f_{n}\right\rangle$ is shown in Figure 13 in log-linear and log-log scales at large strains (the data are cumulated from several snapshots in the residual state) for all the simulations. As usually observed in the literature $[38,13,35,5$, $42,8,9]$, the number of forces above the mean value $\left\langle f_{n}\right\rangle$ falls off exponentially whereas the number of forces below the mean value varies as a power-law:

$$
P\left(f_{n}\right) \propto\left\{\begin{array}{lll}
e^{-\alpha\left(1-f_{n} /\left\langle f_{n}\right\rangle\right)} & \text { for } & f_{n}>\left\langle f_{n}\right\rangle \\
\left(f_{n} /\left\langle f_{n}\right\rangle\right)^{\beta} & \text { for } & f_{n}<\left\langle f_{n}\right\rangle
\end{array}\right.
$$

where $\alpha$ and $\beta$ are the exponents. As we can observe, the pdf of the forces in each samples collapse to the same curve given precisely by (25). This shows that the inhomogenous character of the force distribution chain in granular media is not affected by the number of subdomains used for the solver.

The genuine organization of contact forces in granular media was first analyzed by Radjaï et al. by means of contact dynamics simulations for packings of circular and spherical particles [40]. The most important result was that the contact network can be decomposed unambiguously into two subnetworks named "weak" and "strong" networks with complementary mechanical properties. More precisely, stronger forces chains are propped by large number of weak 

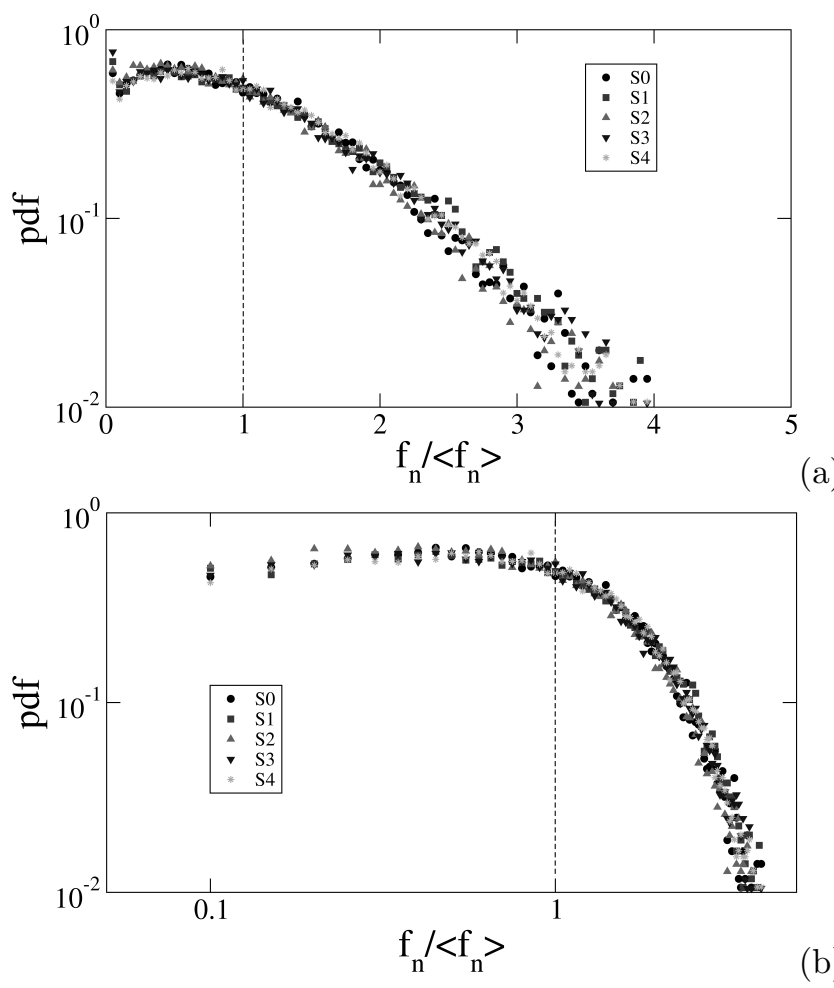

Figure 13: Probability distribution function of normal forces $f_{n}$, normalized by the average normal force $\left\langle f_{n}\right\rangle$ in log-linear (a) and log-log (b) plots for all the tested decompositions. 
contacts, so that the shear stress is almost totally sustained by the strong contact network. This is well illustrated also in Figure 10 where strong forces are plotted in black whereas weak forces are plotted in red. We see that strong forces are mainly vertical (along the principal shear direction) whereas weak forces are, in average, perpendicular to the direction of shear. Data are also in good qualitative agreement with this feature, without much influence of the number of subdomains.

\section{Time consuming analysis of the NSCDD Se- quential Multidomain implementation}

The issue of this section is to estimate the CPU time gain that we can expect from a multiprocessing implementation of the NSCDD algorithm on a distributed memory architecture. We present at first the implementation and the parameters chosen before to an analyse of the numerical tendencies of the proposed method.

\subsection{Sequential Multidomain implementation and chosen parameters}

A Sequential Multidomain implementation of NSCDD algorithm has been performed on the LMGC90 software to study the influence of a domain decomposition on the biaxial test presented above. To do so, the sequential LMGC90 database has been duplicated, according to the number of subdomains considered, to mimic the behavior of a multiprocessing environment. This approach allows to separate the topic of physical validity of the solution given by the proposed domain decomposition method from the technical aspects of MPI implementation.

For the simulations we selected $n_{g s}=1$ (cf. Algorithm 1), which means that one NLGS iteration in subdomains is always followed by an interface resolution, consistently with the study of the influence of $n_{g s}$ parameter on the convergence of the NSCDD algorithm reported in [23].

The cumulative elapsed time and timers of the main steps of the NSCDD algorithm for samples $S 0, S 2$ and $S 4$ is given in Table 1, for the Biaxial test presented above, and performed over $25010^{3}$ time steps. The various stages are classified as:

- White: generic stages of NSCD algorithm which may be parallelized,

- Light-gray: stages introduced by the domain decomposition which may be parallelized (intermediary routines between generic stages of NSCD algorithm and specific stages of the NSCDD one).

- Dark-gray: stages of the NSCDD algorithm which must be done sequentially (if a slave/master communication scheme is presupposed) and implies exchanges between processors. 


\begin{tabular}{|c|c|c|c|c|c|c|}
\hline \multirow{2}{*}{$\begin{array}{l}\text { Main } \\
\text { stages/Samples }\end{array}$} & \multicolumn{2}{|c|}{$S 0$} & \multicolumn{2}{|c|}{$S 2$} & \multicolumn{2}{|c|}{$S 4$} \\
\hline & $\%$ & $\begin{array}{l}\text { Elapsed } \\
\text { time }\end{array}$ & $\%$ & $\begin{array}{l}\text { Elapsed } \\
\text { time }\end{array}$ & $\%$ & $\begin{array}{l}\text { Elapsed } \\
\text { time }\end{array}$ \\
\hline \multicolumn{7}{|c|}{ Preprocessing } \\
\hline $\begin{array}{l}\text { Domain parti- } \\
\text { tioning and rough } \\
\text { detection }\end{array}$ & $2 \%$ & $410^{3} \mathrm{~s}$ & $2 \%$ & $410^{3} \mathrm{~s}$ & $2 \%$ & $510^{3} \mathrm{~s}$ \\
\hline Fine detection & $5 \%$ & $1410^{3} \mathrm{~s}$ & $6 \%$ & $1410^{3} \mathrm{~s}$ & $8 \%$ & $1910^{3} \mathrm{~s}$ \\
\hline $\begin{array}{l}\text { NLGS prepro- } \\
\text { cessing }\end{array}$ & $32 \%$ & $8310^{3} \mathrm{~s}$ & $25 \%$ & $5710^{3} \mathrm{~s}$ & $16 \%$ & $4110^{3} \mathrm{~s}$ \\
\hline \multicolumn{7}{|c|}{ NSCDD iterations } \\
\hline Compute $\bar{v}_{E}^{d}\left(F_{\Gamma}\right)$ & $0 \%$ & $010^{3} s$ & $4 \%$ & $910^{3} s$ & $7 \%$ & $1710^{3} s$ \\
\hline NLGS iterations & $50 \%$ & $13110^{3} \mathrm{~s}$ & $42 \%$ & $10110^{3} \mathrm{~s}$ & $40 \%$ & $10010^{3} \mathrm{~s}$ \\
\hline Compute $A_{\Gamma E} \bar{V}_{E}$ & $0 \%$ & $010^{3} s$ & $9 \%$ & $2110^{3} s$ & $13 \%$ & $3310^{3} \mathrm{~s}$ \\
\hline Interface problem & $0 \%$ & $010^{3} \mathrm{~s}$ & $1 \%$ & $210^{3} \mathrm{~s}$ & $2 \%$ & $410^{3} s$ \\
\hline $\begin{array}{l}\text { Check conver- } \\
\text { gence }\end{array}$ & $1 \%$ & $210^{3} \mathrm{~s}$ & $1 \%$ & $110^{3} \mathrm{~s}$ & $1 \%$ & $110^{3} s$ \\
\hline \multicolumn{7}{|c|}{ Updates and outputs } \\
\hline Update positions & $9 \%$ & $2310^{3} s$ & $9 \%$ & $2110^{3} s$ & $10 \%$ & $2610^{3} s$ \\
\hline Write files & $2 \%$ & $610^{3} \mathrm{~s}$ & $2 \%$ & $510^{3} \mathrm{~s}$ & $2 \%$ & $510^{3} \mathrm{~s}$ \\
\hline Total & $100 \%$ & $26210^{3} \mathrm{~s}$ & $100 \%$ & $23510^{3} \mathrm{~s}$ & $100 \%$ & $25110^{3} s$ \\
\hline
\end{tabular}

Table 1: Percentage and absolute elapsed time in the main stages of the NSCDD algorithm related to samples $S 0, S 2$ and $S 4$.

\subsection{Analysis of the main referenced stages}

"Domain partitioning and rough detection" (cf Table 1). As presented above, the proposed domain partitioning leans on contact distribution among the subdomains. More precisely, the considered contacts are those roughly selected according to a box method referenced as the "rough detection". The elapsed time for each samples is quite similar, a small increase is observed only for S4. In our simulations this phase is performed every 10 time steps leading to a very small contribution to the overall computation time.

"Fine detection" and "Update positions". The increase in the elapsed time related to those stages arises from the number of particles in samples, which increases with the number of subdomains $\left(n_{s d}\right)$ due to the duplication of interface grains, as illustrated in Table 2.

"NLGS preprocessing". This routine stores every extra-diagonal block matrix of the Delassus operator $\left(W_{\alpha \beta}, \alpha \neq \beta\right)$. Its running time decreases according to the number of subdomains. Indeed the duplication of the interface grains in the neighboring subdomains reduces the number of the adjacent contacts (and so the number of $W_{\alpha \beta}$, cf. Table 2). This phenomenon also explains the similar behavior of "NLGS iterations" stage.

"Compute $\bar{v}_{E}^{d}\left(F_{\Gamma}\right)$ " and "Compute $A_{\Gamma E} \bar{V}_{E}$ ". As expected, the elapsed time increases regularly according to the size of the interface.

"Check Convergence". A similar elapsed time is observed for each samples. 


\begin{tabular}{|l||c|c|c|c|c|}
\hline & $S 0$ & $S 1$ & $S 2$ & $S 3$ & $S 4$ \\
\hline $\begin{array}{l}\text { Total number of } \\
\text { NSCDD iterations }\end{array}$ & $164.510^{5}$ & $164.110^{5}$ & $164.110^{5}$ & $166.410^{5}$ & $166.010^{5}$ \\
\hline $\begin{array}{l}\text { Number of inter- } \\
\text { face grains }\end{array}$ & 0 & 117 & 104 & 207 & 227 \\
\hline Number of $W_{\alpha \beta}$ & $41810^{3}$ & $38010^{3}$ & $34110^{3}$ & $36610^{3}$ & $30310^{3}$ \\
\hline
\end{tabular}

Table 2: Total number of NSCDD iterations, mean number of interface particles and mean number of extra-diagonal block matrices of the Delassus operator $\left(W_{\alpha \beta}\right)$ over the $25010^{3}$ time steps of the processes related to samples $S 0, S 1$, $S 2, S 3$ and $S 4$.

The numerical monitoring shows that the time consuming stages may be parallelized whereas the sequential stages requires at most $5 \%$ of the total CPU time in our study. Moreover, even for a Sequential Multidomain implementation, the total elapsed time may be reduced when using several subdomains in comparison with a single subdomain, in spite of the increase of the interface size (in terms of unknowns and equations). This is due to the simultaneous decrease of $W$ size (from $41810^{3}$ to $30310^{3} W_{\alpha \beta}$, cf. Table 2). However, the expected gain from MPI implementation may be quite different because of the potentially expensive exchanges between processors.

\section{Conclusion}

The present work gives a new illustration of the ability to use a domain decomposition method coupled with the nonsmooth contact dynamics approach for dealing with large-scale dense granulates. The proposed approach is as close as possible to the standard nonoverlapping DDM for large-scale linear problems, more precisely the FETI approach. As the interfaces are made of grains, the features of the interface matrix has been systematically studied, for example when a grain belongs to more than two subdomains. A mechanical analysis of a biaxial test exemplifies the relevancy of the results in spite of the chaotic behavior of such a system with a large multiplicity of solutions. The solutions may depend locally on the substructuring procedure but the global behavior of the granular medium is preserved. We rediscover the sensitivity, with respect to the discretization, of a ductile material involving localization effects such as shear bands, of plastic nature, that it is modeled by finite elements or by discrete elements. However the discrete elements are not determined by a discretization process but imposed by the microstructure. Moreover the forthcoming behavior is not strongly oriented by the early localization because of the appearance and the vanishing of multiple different shear bands in dry granulates.

The numerical efficiency, especially the scalability, is recovered if a single NLGS iteration is performed in each subdomain in the first stage of the algorithm [23]. It is not necessary to iterate many times in the first stage because the second stage, characterized by the quasi-diagonal interface matrix, does not transfer long-distance correlation through subdomains. Likewise it is not possible to improve the convergence by adding a coarse problem as in classical computational structural mechanics based on the finite element method. Before extracting a macro-homogenized model from the interface problem it is neces- 
sary to enrich this interface problem. Such an approach has been developed in [4] from a theoretical and semi-analytical point of view. As a conclusion of this study the convergence of the so obtained algorithm does not seem to be significantly accelerated whereas the computational cost of the second solution stage strongly increases. Consequently we propose now to develop, not a nonsmooth solver on a single time step but a multiscale time integration scheme over a time interval for granular systems. The principle would be to combine an explicit linear prediction of the interface forces and an implicit correction of the contact impulses inside the subdomains.

Finally the main drawback of the NSCD approach is the possible indetermination of the contact impulses generated by severe kinematic constraints, especially for dense granular systems. This is conveyed in the singularity of the Delassus matrix whose null space represents the self-equilibrated impulse networks. To overcome this indetermination, from an algorithmic viewpoint, represents an important challenge. To conceive a time integration scheme during a process requires to tackle this topic.

Acknowledgements. We warmly thank F. Radjaï for useful and stimulating discussions on the physics and micromechanics of granular materials and $\mathrm{F}$. Dubois for help with numerical developments. This work was partly supported by OSEO, FEDER and the region of Languedoc-Roussillon (Degrip project).

\section{References}

[1] V. Acary. Contribution à la modélisation mécanique et numérique des édifices maçonnés. PhD thesis, Université de la Méditerranée - Aix-Marseille II, 2001.

[2] V. Acary and M. Jean. Numerical simulation of monuments by the contact dynamics method. In DGEMN-LNEC-JRC, editor, Monument-98, Workshop on seismic perfomance of monuments, pages 69-78, Lisbon, Portugal, 1998. Laboratório Nacional de engenharia Civil (LNEC), Lisboa, Portugal.

[3] P. Alart and D. Dureisseix. A scalable multiscale LATIN method adapted to nonsmooth discrete media. Computer Methods in Applied Mechanics and Engineering, 197(5):319-331, 2008.

[4] P. Alart, D. Iceta, and D. Dureisseix. A nonlinear domain decomposition formulation with application to granular dynamics. Computer Methods in Applied Mechanics and Engineering, 205-208(0):59 - 67, 2012. Special Issue on Advances in Computational Methods in Contact Mechanics dedicated to the memory of Professor J.A.C. Martins.

[5] S. J. Antony. Evolution of force distribution in three-dimensional granular media. Phys. Rev. E, 63:011302, 2001.

[6] E. Azéma, N. Estrada, and F. Radjaï. The effect of the angularity of particles on the mechanical response of sheared granular packings. Submitted to Physical Reviews E, 2011.

[7] E. Azéma and F. Radjaï. Stress-strain behavior and geometrical properties of packings of elongated particles. Phys. Rev. E, 81:051304, 2010. 
[8] E. Azéma, F. Radjaï, R. Peyroux, and G. Saussine. Force transmission in a packing of pentagonal particles. Phys. Rev. E, 76:011301, 2007.

[9] E. Azéma, F. Radjaï, and G. Saussine. Quasistatic rheology, force transmission and fabric properties of a packing of irregular polyhedral particles. Mechanics of Materials, 41:721-741, 2009.

[10] M. Barboteu, P. Alart, and M. Vidrascu. A domain decomposition strategy for nonclassical frictional multi-contact problems. Computer Methods in Applied Mechanics and Engineering, 190:4785-4803, 2001.

[11] C. Bernardi, Y. Maday, and A. T. Patera. A new nonconforming approach to domain decomposition: The mortar element method. In H. Brezzi, editor, Nonlinear partial differential equations and their applications, pages 13-51. Paris, 1994.

[12] B. Cambou, M. Jean, and F. Radjaï, editors. Micromechanics of Granular Materials. ISTE Ltd. and Jhon Wiley and Sons Inc., 2009.

[13] S. N. Coppersmith, C. Liu, S. Majumdar, O. Narayan, and T. A. Witten. Model for force fluctuations in bead packs. Phys. Rev. E, 53(5):4673-4685, 1996.

[14] P. A. Cundall and O. D. L. Stack. A discrete numerical model for granular assemblies. Geotechnique, 29(1):47-65, 1979.

[15] Y.-H. De Roeck, P. Le Tallec, and M. Vidrascu. A domain decomposed solver for nonlinear elasticity. Computer Methods in Applied Mechanics and Engineering, 99:187-207, 1992.

[16] F. Dubois, M. Jean, M. Renouf, R. Mozul, A. Martin, and M. Bagneris. LMGC90. In 10e colloque national en calcul des structures - CSMA2011, Giens, France, 2011.

[17] D. Dureisseix. Une approche multi-échelles pour des calculs de structures sur ordinateurs à architecture parallèle. PhD thesis, ENS de Cachan, 1997.

[18] D. Dureisseix and C. Farhat. A numerically scalable domain decomposition method for the solution of frictionless contact problems. International Journal for Numerical Methods in Engineering, 50(12):2643-2666, 2001.

[19] C. Farhat, M. Lesoinne, P. Le Tallec, K. Pierson, and D. Rixen. FETI-DP: A dual-primal unified FETI method - Part I: A faster alternative to the two-level FETI method. International Journal for Numerical Methods in Engineering, 50(7):1523-1544, 2001.

[20] GDR-MiDi. On dense granular flows. Eur. Phys. J. E, 14:341-365, 2004.

[21] T. M. P. Hoang, P. Alart, D. Dureisseix, and G. Saussine. A domain decomposition method for granular dynamics using discrete elements and application to railway ballast. Annals of Solid and Structural Mechanics, 2011. To appear. 
[22] T. J. R. Hughes and J. Winget. Finite rotation effects in numerical integration of rate constitutive equations arising in large deformation analysis. Computer Methods in Applied Mechanical Engineering, 15(12):1862-1867, 1980.

[23] D. Iceta, D. Dureisseix, and P. Alart. Mixed versus impulse-oriented domain decomposition method for granular dynamics. European Journal of Computational Mechanics, 18(5-6):429-443, 2009. hal-00447176.

[24] M. Jean. The non-smooth contact dynamics method. Computer Methods in Applied Mechanics and Engineering, 177:235-257, 1999.

[25] F. Jourdan, P. Alart, and M. Jean. A Gauss-Seidel like algorithm to solve frictional contact problems. Computer Methods in Applied Mechanics and Engineering, 155(1-2):31-47, 1998.

[26] H. B. Khenous, P. Laborde, and Y. Renard. Mass redistribution method for finite element contact problems in elastodynamics. European Journal of Mechanics - A/Solids, 27(5):918 - 932, 2008.

[27] N. Kruyt and L. Rothenburg. Kinematic and static assumptions for homogenization in micromechanics of granular materials. Mechanics of Materials, 36(12):1157-1173, Dec. 2004.

[28] P. Ladevèze, A. Nouy, and O. Loiseau. A multiscale computational approach for contact problems. Computer Methods in Applied Mechanics and Engineering, 191:4869-4891, 2002.

[29] P. Le Tallec. Domain-decomposition methods in computational mechanics. Computational Mechanics Advances, 1(2):121-220, 1994. North-Holland.

[30] M. Lesoinne. A FETI-DP corner selection algorithm for three-dimensional problems. In 14th International Conference on Domain Decomposition Methods, Mexico, 2002.

[31] J. Mitchell and K. Soga. Fundamentals of Soil Behavior. Wiley, New-York, NY, 2005.

[32] J. Moreau. Indetermination in the numerical simulation of granular systems. In Powders and Grains 2005, volume 1, pages 109-112. Balkema, 2005.

[33] J. J. Moreau. Numerical investigation of shear zones in granular materials. In D. E. Wolf and P. Grassberger, editors, Friction, Arching, Contact Dynamics, pages 233-247, Singapore, 1997. World Scientific.

[34] J. J. Moreau. Numerical aspects of sweeping process. Computer Methods in Applied Mechanics and Engineering, 177:329-349, 1999.

[35] D. M. Mueth, H. M. Jaeger, and S. R. Nagel. Force distribution in a granular medium. Phys. Rev. E., 57(3):3164-3169, 1998.

[36] S. Nineb, P. Alart, and D. Dureisseix. Domain decomposition approach for nonsmooth discrete problems, example of a tensegrity structure. Computers and Structures, 85(9):499-511, 2007. 
[37] F. Radjaï and F. Dubois, editors. Discrete-Element Modeling of Granular Materials. ISTE Ltd. and John Wiley and Sons Inc., 2011.

[38] F. Radjaï, M. Jean, J. Moreau, and S. Roux. Force distributions in dense two dimensional granular systems. Phys. Rev. Letter, 77:274-277, 1996.

[39] F. Radjaï, H. Troadec, and S. Roux. Key features of granular plasticity. In S. Antony, W. Hoyle, and Y. Ding, editors, Granular Materials: Fundamentals and Applications, pages 157-184, Cambridge, 2004. RS.C.

[40] F. Radjaï, D. E. Wolf, M. Jean, and J. J. Moreau. Bimodal character of stress transmission in granular packings. Physical Review Letters, 80(1):6164, 1998.

[41] M. Renouf and P. Alart. Conjugate gradient type algorithms for frictional multi-contact problems: Applications to granular materials. Computer Methods in Applied Mechanics and Engineering, 194:2019-2041, 2004.

[42] V. Richefeu, E. Azéma, F. Radjaï, and M. S. El Youssoufi. Force distribution in cohesive and non cohesive granular media. Powder Technology, 190:258263, 2009.

[43] D. Rixen and C. Farhat. A simple and efficient extension of a class of substructure based preconditioners to heterogeneous structural mechanics problems. International Journal for Numerical Methods in Engineering, 44(4):489-516, 1999.

[44] L. Rothenburg and R. J. Bathurst. Analytical study of induced anisotropy in idealized granular materials. Geotechnique, 39:601-614, 1989.

[45] B. Saint-Cyr, J.-Y. Delenne, C. Voivret, F. Radjaï, and P. Sornnay. Rheology of granular materials composed of nonconvex particles. Accepted in Physical Reviews E, 2011.

[46] G. Saussine, C. Cholet, P.-E. Gautier, F. Dubois, C. Bohatier, and J. J. Moreau. Modelling ballast behaviour under dynamic loading, part 1: A 2D polygonal discrete element method approach. Computer Methods in Applied Mechanics and Engineering, 195(19-22):2841-2859, 2005.

[47] L. Staron and F. Radjaï. Friction versus texture at the approach of a granular avalanche. Phys. Rev. E, 72:1-5, 2005.

[48] H. Troadec, F. Radjaï, S. Roux, and J.-C. Charmet. Model for granular texture with steric exclusions. Phys. Rev. E, 66:041305, 2002.

[49] C. Voivret, F. Radjaï, J.-Y. Delenne, and M. S. El Youssoufi. Space-filling properties of polydisperse granular media. Phys. Rev. E, 76:021301-1, 021301-12, 2007.

[50] C. Voivret, F. Radjaï, J.-Y. Delenne, and M. S. El Youssoufi. Multiscale force networks in highly polydisperse granular media. Phys. Rev. Lett., 102:178001, 2009. 